\title{
Simulation of GaAs p-i-n Diodes
}

\author{
A. GOPINATH, SENIOR MEMBER, IEEE, AND H. ATWATER, SENIOR MEMBER, IEEE
}

\begin{abstract}
GaAs p-i-n diodes have been modeled using simulation, and the results have been compared to experiment. The simulations predict that with a lifetime of the carriers of $10^{-7} \mathrm{~s}$, devices that have good i-layer modulation may be built. This is in agreement with currently available commercial devices.
\end{abstract}

\section{INTRODUCTION}

G aAs p-i-n diodes have been used as photodetectors for some considerable time, but their appearance as a microwave switch element is comparatively recent [1][3]. The problem perceived as preventing their application in this context was the minority-carrier lifetime of about $10^{-8} \mathrm{~s}$ and hence the ambipolar diffusion length of about $3 \mu \mathrm{m}$. Thus, a diode with an i-layer of $10 \mu \mathrm{m}$ would not be easily modulated at reasonable current densities. However, lifetimes now are in the range of $10^{-7} \mathrm{~s}$, and thus commercial diodes with i-layers in the range of 3 to $30 \mu \mathrm{m}$ are available.

While the silicon $p-i-n$ diode has been examined in great detail [4]-[6], the GaAs device is only now being investigated. The simple theory for silicon devices where the mobility ratio of electrons to holes is approximately 3 , and the ratio of the i-layer thickness to diffusion length is less than unity, cannot be used for GaAs diodes, without substantial modification. The present paper reports on the results of an investigation on these devices, using numerical simulation and measurements. In these devices, the $\mathrm{i}$-layer is low-doped $\mathrm{p}^{-}$or $\mathrm{n}^{-}$, and for this investigation, the layer was assumed to be low-doped $\mathrm{n}^{-}$type. The doping level was chosen so that this i-layer is entirely depleted under zero-bias condition due to the built-in junction potential. Thus, the diode equivalent circuit of the device at zero or negative bias takes the form of a capacitor, with a shunt resistor to represent the leakage current and a resistance in series with this parallel circuit to account for the contact resistance (see Fig. 1). The capacitance in this state may be calculated quite simply with a knowledge of the diode dimensions for a uniformly doped diode. The shunt and series resistors are dependent on the i-layer material and the fabrication technique used. The equivalent circuit under zero or reverse bias in this case is shown in Fig. 1.

Manuscript received August, 24, 1987; revised November 17, 1987. This work was sponsored by the Department of the Army.

A. Gopinath was with the MIT Lincoln Laboratory, Lexington, MA 02173. He is now with the Department of Electrical Engineering, University of Minnesota, Minneapolis, MN 55455.

H. Atwater was with the MIT Lincoln Laboratory, Lexington, MA 02173. He is now with the Naval Postgraduate School, Monterey, CA 93940.

IEEE Log Number 8718964.

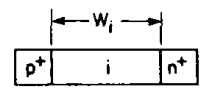

(a)

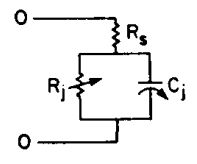

(b)

Fig. 1. (a) Structure of $p-i-n$ diode. (b) Equivalent circuit of $p-i-n$ diode.

The forward bias case is much more complex, and the investigation has examined the parameters contributing to the equivalent circuit components under this condition. The capacitance in this case is due to charge storage in the i-layer in Fig. 1. The shunt resistance is that of the $i-$ layer modulated by the carrier injection from the $\mathrm{p}^{+}$and $\mathrm{n}^{+}$regions. For microwave switch applications, this resistance should be as low as possible, since it is the major factor in the insertion loss to isolation ratio. Analysis of the diode under all forward bias conditions is difficult, and only the case with very high injection [7], [8] may be treated. Under this condition, the hole and electron densities may be assumed to be equal almost everywhere in the diode, and thus the electron and hole density distribution through the diode may be estimated. From this, the voltage drop across the diode may be calculated, from which diode forward resistance may be obtained. We do not include this analysis, but interested readers may refer to Ghandhi [7]. However, the approximations in this analysis, while providing a result for the high-injection condition, do not pertain to all the bias conditions in the device. Thus, numerical analysis provides the answers for the whole range of injection levels.

The results of this numerical investigation are for the cases where the lifetime of the carriers is fixed and device length varied, and also where the device length is fixed and the carrier lifetime is varied. They show that when the lifetime of the carriers falls, and thus, the ratio of diode length to diffusion length is much greater than about 3 , the operation of the device with low forward resistance requires a large voltage drop across the i-region and may not be satisfactory. Comparison with experimental measurements on commercial diodes confirms the trends obtained here.

\section{Numerical Simulations and Results}

As the whole range of bias conditions cannot be easily analyzed, numerical simulation of the diode has been car- 
ried out. This simulation follows the usual solution of the Poisson and the continuity equations for electrons and holes simultaneously using the Scharfetter-Gummel approximation, which is the "up-stream" formulation for current. The finite-difference and finite-element methods in one dimension lead to identical equations for solution purposes. The low-injection case may be handled using the Gummel scheme of separation of the solutions of the Poisson and the continuity equations. However, Newton's method was used here since both the low- and highinjection cases are to be solved. The choice of the parameter to prevent overshoot in Newton's method followed that of Banks and Rose [9] (see also [10]), with additional upper and lower limits placed on the method. Convergence was very rapid (less than 10 iterations) with proper choice of the overshoot parameter, but a more conservative estimate of this parameter resulted in convergence, even with very high injection within 50 iterations at most. As in all simulations, the equations have been scaled in the manner discussed by Selberherr [11].

The choice of the structure analyzed was governed by various considerations including time-dependent solutions (which will be reported elsewhere). The structure has $\mathrm{p}^{+}$and $\mathrm{n}^{+}$and regions, a central $\mathrm{n}^{-}$region, with tapered changes between the $\mathrm{p}^{+}$and $\mathrm{n}^{-}$region and the $\mathrm{n}^{-}$ and $\mathrm{n}^{+}$region, both within $0.2 \mu \mathrm{m}$. The $\mathrm{p}^{+}$and $\mathrm{n}^{+}$doping densities were chosen to be $10^{17}$ per $\mathrm{cm}^{3}$ with complete ionization, which, in general, is low for these devices, but the results are not unduly affected by this choice. The ilayer ( $n^{-}$region) width was chosen as $10 \mu \mathrm{m}$ for one set of computations, and other widths were used for a second set. The $\mathbf{n}^{-}$density for all of the diodes examined was $10^{13} \mathrm{~cm}^{-3}$ with complete ionization, and this depleted due to the $p^{+}-n^{-}$junction barrier voltage. The recombination terms included single-level traps of the usual form of Shockley-Read-Hall

$$
R_{\tau}=\frac{p n-n_{t}^{2}}{\tau_{p}\left(n+n_{t}\right)+\tau_{n}\left(p+p_{t}\right)} \mathrm{cm}^{-3} / \mathrm{s} .
$$

Auger recombination was assumed to be of the form

$$
R_{A}=r\left(n^{2} p+p^{2} n\right) \text {. }
$$

Radiative recombination was assumed to be given by

$$
R_{r}=\beta n p .
$$

In (1), $\tau_{p}$ was set to be equal to $\tau_{n}$, and this parameter was varied from $10^{-6}$ to $3 \times 10^{-10} \mathrm{~s}$ for the $10-\mu \mathrm{m}$ i-layer diode.

The Auger recombination term only becomes significant at levels of $10^{17} \mathrm{~cm}^{-3}$ and thus was not the control mechanism in these simulations. The radiative term is in general small unless densities reach the high $10^{18} \mathrm{~cm}^{-3}$. The values of $r$ and $\beta$ chosen were $10^{-31}$ and $7.2 \times 10^{-10}$, respectively.

The barrier heights were estimated using the usual formulas for $N_{c}, N_{v}$, using the effective masses as $m_{e}=$ $0.065 m_{0}$ for electrons and $m_{h}=0.5 m_{0}$ for holes, with $E_{g}$ at room temperature of $1.42 \mathrm{eV}$ (see Sze [12]). The ve- locity-field expression for electrons takes the form

$$
v_{e}=\frac{\mu_{0} E+v_{s}\left(\frac{E^{4}}{E_{0}}\right)}{1+\left(E / E_{0}\right)^{4}}
$$

where $E_{0}=4 \times 10^{3} \mathrm{~V} / \mathrm{cm}$ and $\mu_{0} \simeq 5000 \mathrm{~cm}^{2} / \mathrm{V} \cdot \mathrm{s}$. The hole velocity was assumed to vary as

$$
v_{h}=\frac{\mu_{h} E}{1+E / E_{0}}
$$

where $E_{0}=2 \times 10^{4} \mathrm{~V} / \mathrm{cm}$ and $\mu_{h} \simeq 400 \mathrm{~cm}^{2} / \mathrm{V} \cdot \mathrm{s}$.

Einstein's relationship was assumed to hold, and thus the diffusion constant was estimated from these expressions.

Fig. 2(a) and (b) shows a sequence of results from these calculations in which the carrier density distributions and potential are shown at different bias conditions for a diode with an $\mathrm{n}^{-}$layer of $10 \mu \mathrm{m}$, a total length of $10.8 \mu \mathrm{m}, \tau$ of $10^{-9} \mathrm{~s}$ in the $\mathrm{n}^{-}$layer, and $10^{-11} \mathrm{~s}$ in the $\mathrm{n}^{+}$and $\mathrm{p}^{+}$ regions. The current-voltage relationship in these devices provides a summary of all the computed cases; two sets of results are reported here, and we discuss each set in turn. In the first set, the doping density of the device structure was kept constant, and the i-layer ( $n^{-}$type) thickness was varied form 3 to $20 \mu \mathrm{m}$. In this computation, the lifetime was set to $10^{-8} \mathrm{~s}$, and with a hole mobility of $400 \mathrm{~cm}^{2} / \mathrm{V} \cdot \mathrm{s}$; the diffusion length $L_{a}$ is about $3 \mu \mathrm{m}$. Fig. 3 shows the $J-V$ plots for this set of calculations, and we note that the ideality factor at low current densities is close to unity; this factor becomes larger at higher bias values. Note that the current density in the 20 $\mu$ m diode is much lower for the same bias, suggesting that i-layer modulation may be poor for this diode. In this case the ratio of $d / L_{a} \simeq 3$ (note that $2 d=20 \mu \mathrm{m}$ here), which suggests that, when the $d / L_{a}$ ratio exceeds about 2 , ilayer modulation is poor. Note that the i-layer in the 20 $\mu \mathrm{m}$ device is not completely depleted.

The second set of results in Fig. 4 is for a $10-\mu \mathrm{m}$ i-layer for different values of lifetime; $\tau$ varies from $10^{-6}$ to $3 \times$ $10^{-10} \mathrm{~s}$. Thus, the diffusion length varies from a maximum of $30 \mu \mathrm{m}$ to about $0.5 \mu \mathrm{m}$, and the $d / L_{a}$ ratio varies from about 0.16 to about 10 . We note that the same trend is followed as in Fig. 3, and again $d / L_{a}$ ratios of 2 upward $\left(10^{-9}\right.$ and $\left.3 \times 10^{-10} \mathrm{~s}\right)$ show that current densities do not rise far enough for reasonable i-layer modulation. Note that the two sets of curves, while showing similar trends, show differences in detail. The curves in Fig. 4 at the lower lifetimes and thus smaller $d / L_{a}$ ratios, seem to coalesce, whereas there is still an increase in current density at the smaller i-layer widths in Fig. 3.

The slope of the $J-V$ curves at any bias point is the incremental resistance seen by small signal RF voltage.

\section{Measurements}

Comparison between experiment and theory is difficult because the doping profile of the devices is not known. The devices measured are expected to have $\mathrm{p}^{+}$and $\mathrm{n}^{+}$ regions doped to about $10^{18}$ acceptors or donors per cubic 


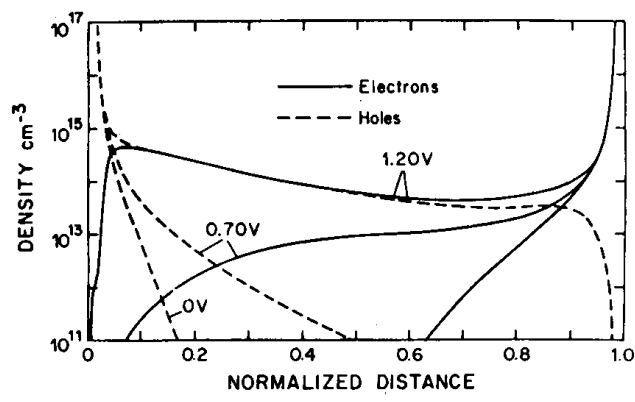

(a)

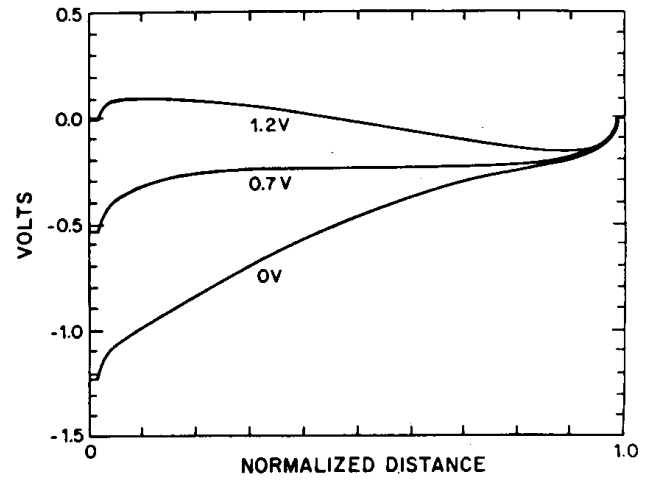

(b)

Fig. 2. Sequence of steady state (a) carrier density and (b) potential distribution for a $10-\mu \mathrm{m}$ i-layer diode, $\tau=10^{-9} \mathrm{~s}$ and density is $10^{13} \mathrm{~cm}^{-3}$ in this region for different bias conditions.

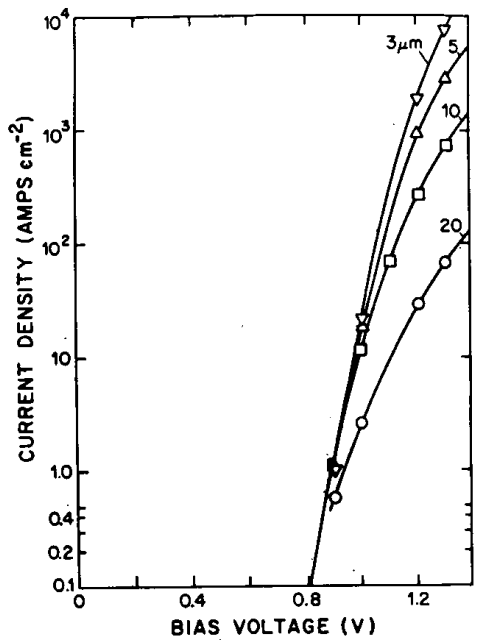

Fig. 3. Current density versus bias voltage for $\mathrm{GaAs}$ p-i-n diode, $\mathrm{i}$-layer density is $10^{13} \mathrm{~cm}^{-3}, \tau=10^{-8} \mathrm{~s}$, for different i-layer widths.

centimeter. The choice of the $\mathrm{n}^{-}$doping for the $\mathrm{i}$-region is also not known, and this could vary from $10^{14}$ per $\mathrm{cm}^{3}$ to lower values, depending on the i-layer thickness. Thus, the built-in potential, calculated at $1.23 \mathrm{~V}$ for the structures considered may not be the correct value for the devices on which measurements were made. Furthermore, the mobility values chosen for the computations may not agree with device values, and this will in turn affect the

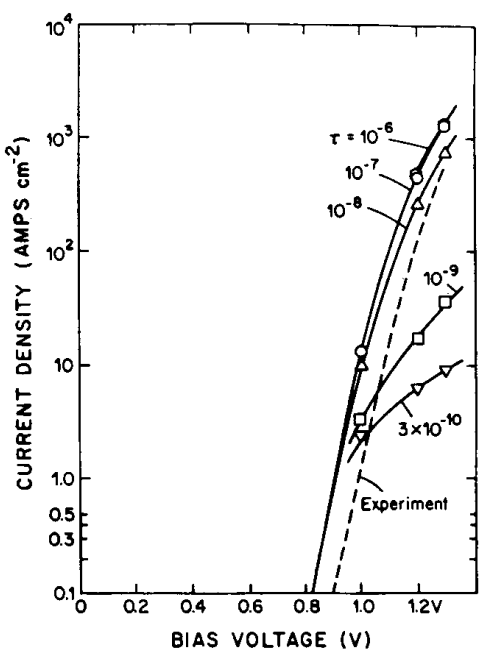

Fig. 4. Current density versus bias voltage for $\mathrm{GaAs}$ p-i-n diode, i-layer density $10^{13} \mathrm{~cm}^{-3}$, width is $10 \mu \mathrm{m}$, for different values of $\tau$.

diffusion coefficients that are used in the calculations. Thus, comparison between experiment and theory would indicate trends, rather than agreement in detail.

In Fig. 4 , the $J-V$ characteristic is plotted for the experimental device, a $10-\mu \mathrm{m}$ i-layer, $100-\mu \mathrm{m}$-diaemeter mesa structure. Note that the slope is almost the same as the computed results for $\tau=10^{-7} \mathrm{~s}$. The shift of the curve with respect to the voltage axis arises from several reasons: the surface depletion effect permits no current to flow in this depleted area and thus decreases the effective area of the diode. The second reason for the shift in the voltage axis is due to the ohmic contact drops, modeled as a series resistance. The third reason is that the built-in potential in the calculations may be smaller by about 40 $\mathrm{mV}$. Thus, with these effects taken into account, the $J-V$ curves show good agreement.

Fig. 5 shows the incremental resistance of this device obtained from the measured $I-V$ characteristics. The slope of the $I-V$ curve has its ideality factor close to unity, and this indicates that the current flow is still not dominated by recombination effects. The modeled curve in Fig. 4 with $\tau$ of $10^{-7} \mathrm{~s}$ is close to the measured curve, and therefore not superposed on it, and shows good agreement with experiment.

Microwave measurements were also performed in which the diode was connected to ground at the end of a 50- $\Omega$ line. Since the resistance values are small, the deembedding of the measurements and the separation of the parasitics are difficult with high accuracy. A length of 50$\Omega$ microstrip line was measured with a short circuit, an open circuit, and a $50-\Omega$ chip resistor. The problem is that the short is not a true short, but a small inductance to ground, and the open is not a true open, but a small capacitance to ground. In the process, however, the wire bond is modeled into the parameters for this microstrip line, but the other parasitics are neglected. With the line parameters known, the devices are bonded-in in turn, measured, and de-embedded. The resistance values are 


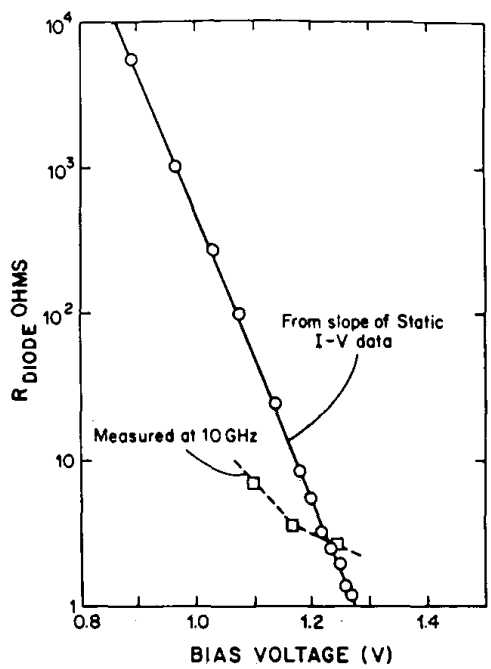

Fig. 5. GaAs p-i-n diode measured forward resistance values for different bias from the slope of the $I-V$ curve and also from microwave measurement at $10 \mathrm{GHz}$.

plotted in Fig. 5 and show broad agreement with theory $\mathrm{dc}$ results. The difference between the dc and RF data may be attributed to the fall in resistance discussed by Caverly and Hiller [13].

\section{Summary}

Numerical simulations have also been carried out for the GaAs p-i-n diode. Experimental $J-V$ characteristics and forward resistance show good agreement with theory. The measured diodes show that the minority-carrier lifetime is about $10^{-7} \mathrm{~s}$, allowing devices in the range of 3to $30-\mu \mathrm{m}$ i-layer thickness to be built .

\section{REFERENCES}

[1] C. Barratt, A. Christou, N. Jansen, R. E. Neidert, M. L. Ruess, Jr., C. W. Young, "New GaAs PIN diodes with lower dissipation loss, faster switching speed at lower drive power," in 1983 IEEE Int. Microwave Symp. Dig. (Boston, May 31-June 3, 1983), pp. 507509.

[2] "GaAs PIN diodes," MA/COM Data Sheet for MA-4GP001, MA4GP002, MA-4GP003.

[3] A. Gopinath, "Comparison of GaAs MESFET and GaAs p-i-n diodes as switch elements," IEEE Electron Device Lett., vol. EDL-6, pp. $505-506,1985$.

[4] D. Leenov, "The silicon PIN diode as microwave radar protector at megawatt levels," IEEE Trans. Electron Devices, vol. ED-11, p. 53$61,1964$.

[5] J. Lepoff, "A new PIN diode for UHF-VHF applications," IEEE Trans. Broadcast Telev. Receivers, vol. BTR-17, pp. 10-15, 1971.

[6] J. F. White, Microwave Semiconductor Engineering. New York: Van Nostrand Reinhold, 1982

[7] S. K. Ghandhi, Semiconductor Power Devices: Physics of Operation and Fabrication Technology. New York: Wiley-Interscience, 1977.

[8] R. J. Chaffin, Microwave Semiconductor Devices: Fundamentals and Radiation Effects. New York: Wiley-Interscience, 1973, pp. 182188.

[9] R. E. Banks and D. J. Rose, "Parameter selection for Newton-like methods applicable to non-linear partial differential equation," SIAM J. Numerical Anal., vol. 17, pp. 806-822, 1980.

[10] - "Global approximate Newton methods," Numerical Math., vol. 37. pp. 279-295, 1981.

[11] S. Selberherr, Analysis and Simulation of Semiconductor Devices. Vienna: Springer-Verlag, 1984, p. 142.

[12] S. M. Sze, Physics of Semiconductor Devices, 2nd ed. New York: Wiley, 1981, pp. 17-19.

[13] R. H. Caverly and G. Hiller, "Microwave resistance of gallium arsenide and silicon $\mathrm{p}$-i-n diodes," in IEEE MTT-S Int. Microwave Symp. Dig. (Las Vegas, NV, June 1987), pp. 591-594.

\section{*}

A. Gopinath, (S'64-M'65-SM'80) photograph and biography not available at the time of publication.

H. Atwater, (S'46-SM'59) photograph and biography not available at the time of publication. 